\title{
A Statistical Classification Method for Hierarchical Irregular Objects
}

\author{
Markus Peura. \\ Helsinki University of Technology \\ Laboratory of Computer and Information Science \\ P.O. Box 2200, FIN-02015 HUT, Finland ${ }^{\star \star}$
}

\begin{abstract}
This paper introduces a method for classifying structured visual objects that appear frequently in meteorological, medical and biological imagery. The focused objects are taken to be highly irregular and composed of subobjects in a hierarchical manner. The approach consists three principal steps. At first, hierarchical objects are detected in an segmented image. Secondly, shape descriptors are used to extract information of the contours of the objects. Finally, a global description for an object is obtained by applying statistical moments. As the goal is to classify natural objects, the most challenging task is to tolerate irregularity present in both spatial and hierarchical levels. Experiments with artificial images show that the method combines succesfully shape descriptors and object hierarchy.
\end{abstract}

\section{Introduction}

Contour irregularity is a pronounced feature of many real-world natural objects. Object hierarchy, as far as it exists, might also be irregular and explicable only by statistical means. Some artificial objects are shown in Fig. 1. In each column, visual similarity between the two objects is obvious. Moreover, one easily gets an intuition of how new instances of each class might look like. The problem is to find a possibly general method for classifying objects of this type.

Sometimes there are no clear, separate "objects" in the imagery - or they remain difficult to extract due to their continous nature, noise or effects of illumination. Depending on the application, there are several other approaches to choose from: histograms, color information or texture.

On the other hand, if objects appear clearly it is possible to utilize image geometry. In this paper, we use shape descriptors as the features of an object. as they complete the topological information in a natural fashion. The shape descriptors measure an object's overall geometrical properties such as elongation, curvature and rectangularity[6, Ch. 6]. Efficiency of some descriptors for irregular forms is discussed in [5].

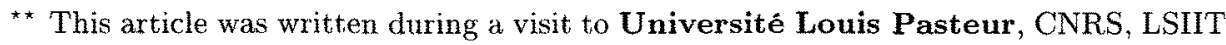
(URA 1871), 7, rue de René Descartes, 67084 Strasbourg, France. 


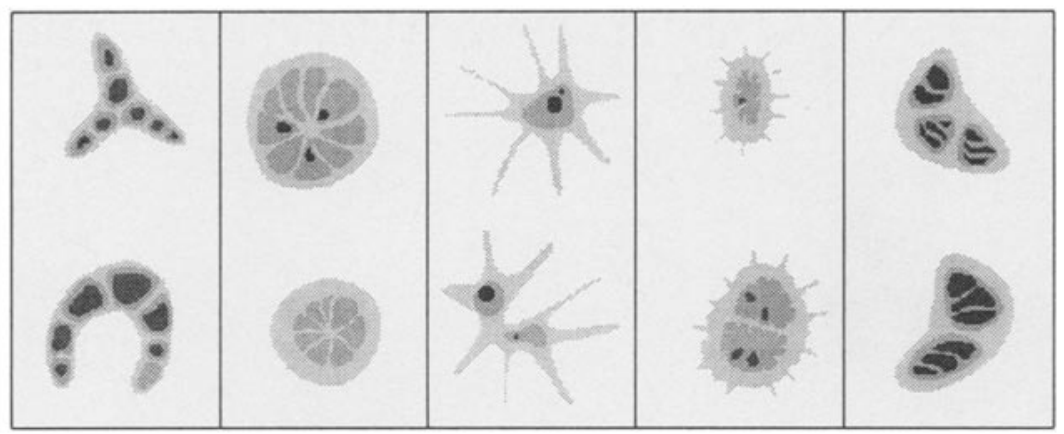

Fig. 1. Examples of irregular hierachical objects.

Hierarchical properties have been recently studied to exploit semantics, the highest level of image understanding. A retrieval system of medical images presented by [3] involved spatial relations of the type "nearby","slightly touching" and "invading". Hierarchy was also applied in calculating distances between the relations.

The emphasis in this paper is on extracting information from both contours and hierarchical structure in a combined fashion. The statistics used to provide an overall descriprion of an object belong to the mathematical family of moments, which was early applied in visual pattern recognition [4]. Related methods have also been adapted directly as shape descriptors [1] and in texture description [2]. Input images are taken to have been preprocessed with some appropriate segmentation algorithm. Nevertheless, the suggested method does not require any additional segment attributes - only forms and spatial organization are considered. If further information exists, it can be flexibly added to the method as proposed in discussion.

This paper is organized as follows. The suggested method is presented in Sec. 2 and consists of definitions of applied object model (2.1), an algorithm for detecting object hierarchy (2.2), the applied object descriptors (2.3) and statistical measures (2.4).

\section{$2 \quad$ Suggested method}

\subsection{Object hierarchy}

A simple visual object is depicted in Fig. 2a. The object consists of segments labeled as 1,2,3 and 4. The background, marked with 0 , can is seen as a special segment - its definition remains application-specific. The simplest interpretation of the object is to regard it as an equal composition of segments $1,2,3$ and 4 .

As far as hierarchy is considered there exists several alternatives for modeling. Clearly, the essential properties are connectivity and nesting. Three different interpretations of hierarchy are illustrated in Fig. 2. The one applied in this 


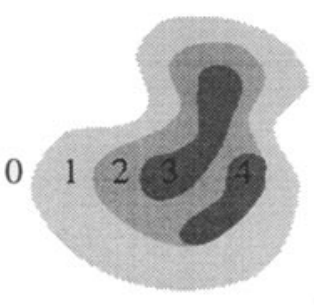

a)

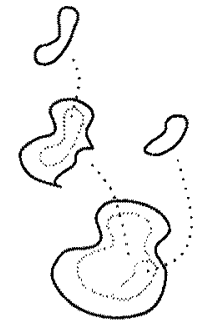

b)

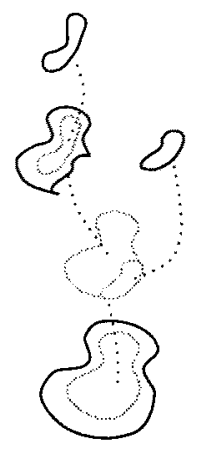

c)

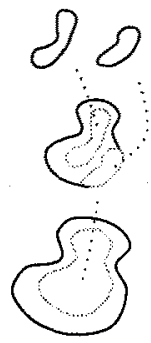

d)

Fig. 2. Alternative interpretations of object hierarchy.

paper is presented in Fig. 2d and applies an additional rule: among connected segments, the segment that has the maximal portion of the common contour is not an object. This concept of ignoring the "dominating" segment is motivated by the intuitive concept of altitude: an object is treated like a set curves on a topographical map. Notice that this rule is not based on topology but on a computational measure. Also the gray tones in Fig. 2a only illustrate the preferred interpretation: as metioned above, colors (or any labels) do not affect the hierarchy.

\subsection{TRIM algorithm}

In this section, an algorithm is presented for constructing a data structure that corresponds to the tree hierarchy described in 2.1. The algorithm, from this on called TRIM (TRee IMage), scans an image in a recursive manner. Each time a nested segment is encountered, a definite border, HardBorder, is drawn around the aggregate of all the connected segments to force traversing the complete object before quitting. This constraint facilitates the bookkeeping: the hierarchy is built one branch at the time. In the hierarchy tree, let us call the nested objects within an object as descendants.

On the other hand, each time a connected segment is found, an one-directional border, SoftBorder is drawn around it: the traversal is allowed to exit the segment but not to return. This ensures that each segment is entered exactly once, thus no multiple instances of the same object will occur. In the hierarchy tree, let us call the connected objects as parallel objects for obvious reasons.

Practically, the algorithm requires an additional image for bookkeeping. Fortunately, HardBorders, SoftBorders and visited pixels can be marked in the same bookkeeping image - eight bits per pixel are sufficient for storing all information involved. The high-level syntax of TRIM is listed in Table 1. An application of the algorithm for the object shown in Fig. 2 is illustrated in Fig. 3. 
Table 1. Top-level description of TRIM algorithm

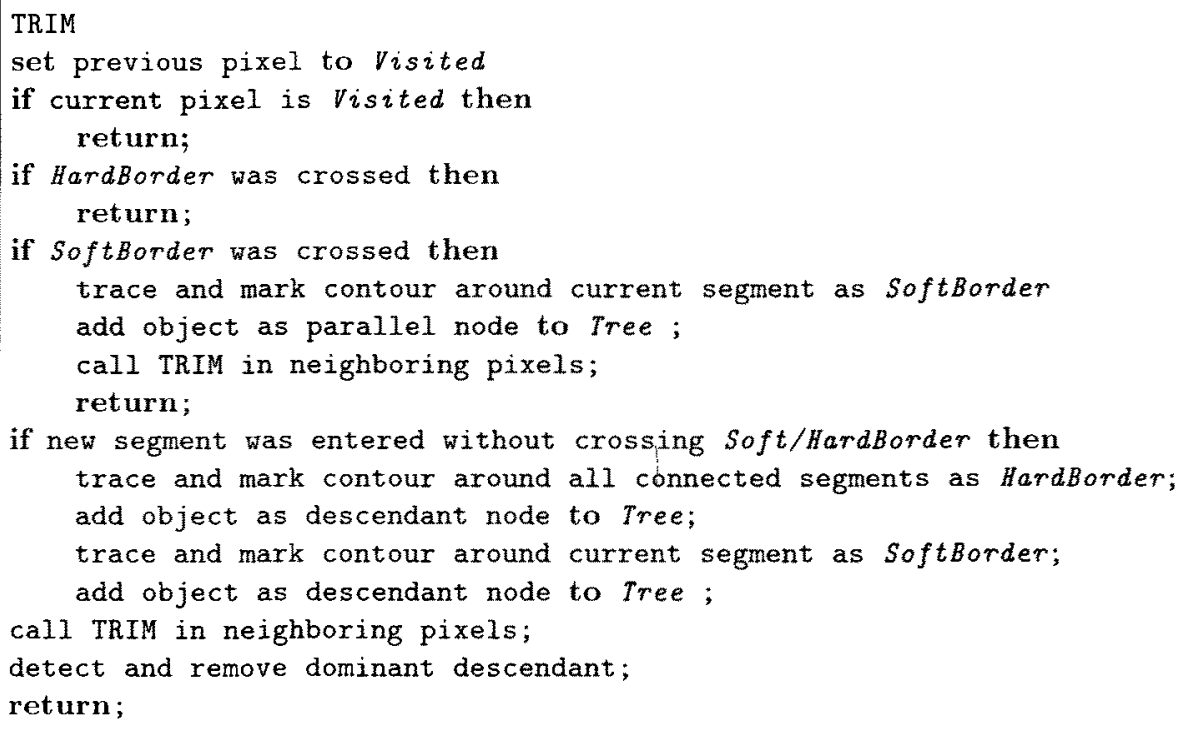

Inside an object (a) the traversal proceeds recursively in all directions. As far as a nested object is encountered (b), HardBorder is drawn around (c) the set of connerted segments. Then, SoftBorder is drawn (d) around the encountered segment. Recursion continues inside the segment but is allowed to pass trough the SoftBorder. For any subsecpucnt comnected segments (e) this procedure will be repeated (f). For new nested sogments $(\mathrm{g})$, the procedure of $(\mathrm{c}-\mathrm{d})$ will be repeated (h). Recursion completes (i) inside the nested segment and continues (j) within the parent object. Before quitting a nested segment, the possible dominant segment is supprined from the tree structure.

a)

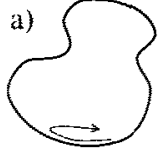

b)
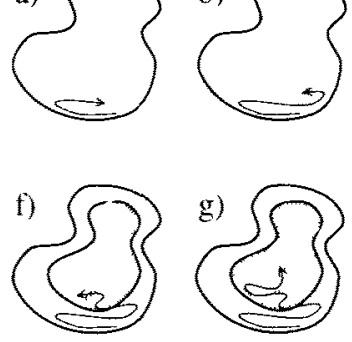

g)

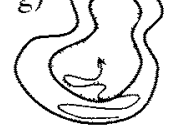

c)
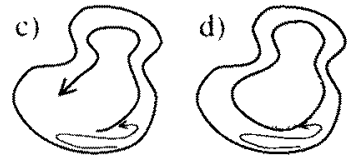

e)
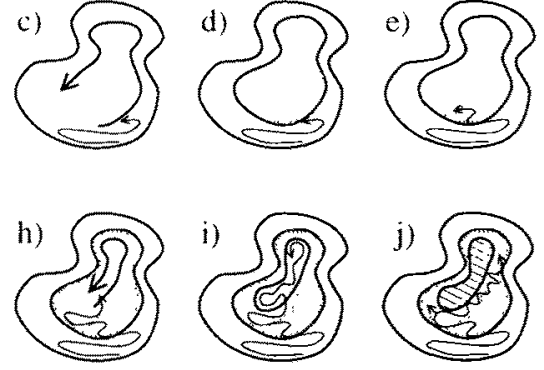

Fig. 3. TRIM algorithm applied to the object shown in Fig. 2a. 


\subsection{Object descriptors}

In order to describe an object one has to carry out measurements. The descendant count $\left(N_{\text {desc }}\right)$, also called the branching factor, is a straightforward topological feature. For example, the object in the upper left corner of Fig. 1 has seven descendants, each of which have one descendant. Proportional area $\left(A_{p r}\right)$ is defined as the area of the object divided by the area of its parent object. Clearly, $A_{p r}$ is not a topological feature but shows practically some inverse correlation with $N_{\text {desc }}$.

In addition, we wish to extract information of object geometry. This is carried out by means of four shape descriptors illustrated in Fig. 4. All these shape descriptors can be calculted in a time linearly proportional to the contour length. Detailed definitions can be found in [5], for example.

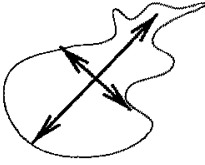

Principal axes

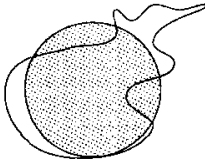

Compactiess

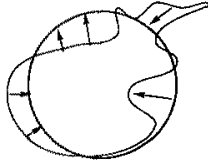

Variance

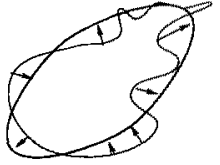

Elliptic variance

Fig. 4. Four simple shape descriptors.

\subsection{Statistical modelling}

As explained in 2.2, TRIM transforms an image to a tree structure. The structure of a tree corresponds to the object topology in the image. All that actually has to be stored in a node is a feature vector consisting of $N_{\text {desc }}, A_{p r}$ and the four shape descriptors - there is no need to store the contour itself. An example of a resulting tree object is depicted in the left hand side of Fig. 5. It is clear that a tree will not preserve all the information present in the original image. However, memory requirements are remarkably decreased, unless an object consists of a large number of small specks.

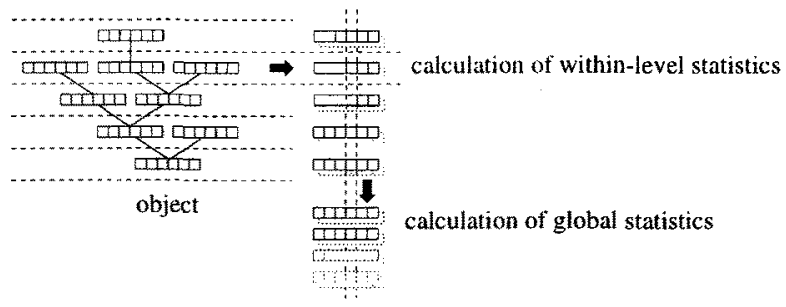

Fig. 5. An object modeled as a tree. Calculation of global statistics. 
For purposes of classification, one has to be able to compare trees. Practically, one applies a codebook, a collection of samples labelled according to correct classes. In our case, a codebook conld consist of trees presented by some appropriate syntax. However, the comparison operation between a given and stored samples might be computationally elaborate. In addition, we desire to make our approach invariant of the hierarchical depth of objects. Thus, instead of matching the tree graphs directly we suggest global statistical measures.

The basic idea is depicted in Fig. 5. The global measures are obtained by applying repeatedly four basic statistics listed in Table 2.

Table 2. Simple statistical moments for variable $x$.

\begin{tabular}{|c|c|c|c|}
\hline$\frac{1}{N} \sum_{t=1}^{N} x_{i}$ & $\frac{1}{N_{n^{2}}} \sum_{i=1}^{N}\left(x_{1}-\mu_{r^{\prime}}\right)^{2}$ & $\frac{1}{N \Sigma, x} \sum_{i=1}^{N} x_{i} i$ & $\frac{l}{N^{2} \Sigma} \sum i=1, l^{2}$ \\
\hline mean $[\mu]$ & nartance $\left.\mid \sigma^{2}\right]$ & centroud $|c|$ & inertia $|\mathcal{I}|$ \\
\hline
\end{tabular}

Given a tree presentation of an object, $\mu$ and $\sigma^{2}$ are calculated at each layer for each element of the feature vector. As the feature vectors consist of six elements, twelve statistics result at each layer. Then, all the statistics $(\mu$, $\sigma^{2}, c$ and $\mathcal{I}$ ) are calculated for the obtained set, resulting in 48 statistics. When calculating the global statistics, the index i equals the height of a layer. Thus, $c$ and $\mathcal{I}$ provide valuable information on how the features are distributed vertically. For example, $\mathcal{I}$ will tell that the compactiness of the objects in the leftmost column of Fig. 1 tends to increase along the levels of the tree hierarchy. $\mathrm{O}_{\mathrm{n}}$ the other hand, the compactness of the objects in the rightmost columm secms relatively constant troughout the levels.

There is no motivation for (alculating the layer-wise $c$ or $\mathcal{I}$, because the? horizontal order of branches is insignificant.

\section{$3 \quad$ Experiments}

The discriminatory power of the proposed method was tested with a set of artificial images consisting of 72 samples: 12 classes, 6 samples in each. The objects shown Fig. 1 belong to this test set and give somo impression of intra- and interclass diversity. To keep the evaluation of results simple, the nearest neighbour $(\mathrm{NN})$ and 3-nearest neighbours algorithm (3NN) were used in the experiments. Implementation of cross-validation was straightforward: every sample, in turn, was picked out from the set and the codebook consisted of the remaining 71 samples. The results for some combinations of features are listed in Table 3 . There are a couple of interesting issues. At first, the within-level variance $\left(\sigma^{2}\right)$ seems to be redundant; without the mean $(\mu)$ reasonable results would not be obtained at. 
all. Secondly, classification using no shape descriptors but hierarchical information only $\left(.4 p\right.$ and $\left.N_{\text {dese }}\right)$ shows suprisingly poor performance. In general, using either shape descriptors or hierarchical information leaves performance around $70 \%$ but their cooperation enhances classification remarkably.

\begin{tabular}{|c|c|c|c|c|c|c|c|c|c|c|c|c|c|}
\hline $\begin{array}{l}\text { shape descriptors } \\
A_{y r} \\
N_{\text {ciess }}\end{array}$ & $\begin{array}{c}x \\
x \\
x\end{array}$ & $\begin{array}{c}x \\
x \\
x\end{array}$ & $x$ & $\begin{array}{l}x \\
x\end{array}$ & $x$ & $\begin{array}{l}x \\
x\end{array}$ & {$\left[\begin{array}{l}x \\
x \\
x\end{array}\right.$} & $\times$ & $\begin{array}{c}x \\
x\end{array}$ & $x$ & $\begin{array}{l}x \\
x \\
\end{array}$ & $\begin{array}{c}x \\
x \\
x \\
\end{array}$ & $\begin{array}{l}x \\
x \\
x\end{array}$ \\
\hline $\begin{array}{l}\text { within-level } \mu \\
\text { within-level } \sigma^{2}\end{array}$ & $x$ & $\times$ & $x$ & $x$ & $x$ & $x$ & $x$ & $\begin{array}{l}x \\
x\end{array}$ & $\begin{array}{l}x \\
x\end{array}$ & \begin{tabular}{|c|} 
\\
$x$ \\
\end{tabular} & \begin{tabular}{|l}
$x$ \\
$x$ \\
\end{tabular} & $\begin{array}{l}x \\
x \\
\end{array}$ & $x$ \\
\hline $\begin{array}{l}\text { global } \mu, \sigma^{2} \\
\text { global } c, \mathcal{I}\end{array}$ & \begin{tabular}{|l}
$x$ \\
$x$ \\
\end{tabular} & \begin{tabular}{|c|} 
\\
$x$ \\
\end{tabular} & \begin{tabular}{|l|}
$x$ \\
$x$ \\
\end{tabular} & $\begin{array}{l}x \\
x\end{array}$ & $\begin{array}{l}x \\
x \\
x\end{array}$ & $\begin{array}{l}x \\
\times \\
\end{array}$ & $x$ & $\begin{array}{l}x \\
x \\
\end{array}$ & \begin{tabular}{|c|}
$x$ \\
$x$ \\
\end{tabular} & \begin{tabular}{|l|}
$x$ \\
$x$ \\
\end{tabular} & \begin{tabular}{|l|}
$x$ \\
$x$ \\
\end{tabular} & $x$ & $\begin{array}{l}x \\
x\end{array}$ \\
\hline $\begin{array}{l}\mathrm{NN} \\
3 \mathrm{NN}\end{array}$ & & & & & & & & & & & & & $\begin{array}{l}47 \\
49\end{array}$ \\
\hline
\end{tabular}

Table 3. Classification percentages for some combinations of features and operators.

\section{Discussion}

A flexible method was presented for recognizing and classifying natural objects. The statistical aproach provided means for treating irregularity and hierarchy in a scale-invariant manner. The experiments showed that the proposed global descriptors are able to extrart central information of the structrural nature of an object. The test set was relatively small, 72 samples, but clearly suggested general applicability of the method.

As the studied objects have tree-like hierarchy, graph matching might be another method for classification. The choice between these two rather different approaches is dependent on the application.

As far as tree hierarchy is considered, visual objects have some special practical restrictions. Inside the contour of an object there is limited space available for subobjects. In natural objects, sizes of subsequent contours tend to change exponentially - for a complicated phenomenon, the resolution runs out in "both ends": some details are smaller than a pixel and some properties are beyond the scope of the whole image. Practically, this means that captured visual objects have relatively simple hierarchy. Forcing more levels to tree hierarchy will naturally not bring any new information: instead of more refined hierarchy (more branching) the tree will only get "streched" as the countours of subsequent objects resemble each other.

Paradoxally, simplicity of visual objects might be a problem when applying statistical approach. For example, the variance is mathematically well-defined for a single sample but its statistical interpretation and significance becomes vague. This problem is visible in the results of the experiments (Table 3); the 
variance seems to produce noise rather than information. This suggests that in classifying a sample, the variance should be used only if the decision, based on the mean only, remains ambiguous.

In the lowest levels of a tree, relatively few nodes contribute to in-level statistics. On the other hand, intuition suggests that the most significant information is located near the root: apparently, the set of artificial test objects could have been recognized well by applying specially designed shape descriptors to the outer contours only. But as mentioned in Sec. 1, the goal was to develop a possibly general approach - which finally showed promising behaviour for the test set containing a liversity of forms.

Practically, success of high-level image understanding requires reliable segmentation, techniques of which were beyond the scope of this paper. For example, due to some unstable segmentation algorithm separate objects might become conneted (or vice versa) producing severe problems in shape description, especially when detecting clongation. Moreover, topology may be distorted if faulty connected objects create closed chains and thus new "irreal" subobjects.

The background and the motivation for this study was in interpretation of meteorological radar images but the general applicability of the proposed method is obvious. Moreover, the molel is directly adjustable to utilize any applicationspecific segment information: existing feature vectors are easily angmented with further elements. This information can be obtained during segmentation or by means of more complex shape descriptors.

\section{Acknowledgement}

The author wishes to thank the Finnish Foundation of Technology Development (Tekniikan Edistämissäätiö) for funding and professor J. Korczak at Louis Pasteur University, Strashourg, for providing research facilities. The weather radar reseach team at the Finnish Meteorological Institute is acknowledged for cooperation.

\section{References}

1. L. Gupta aud M.D. Srinath. Contour sequence moments for the classification of closed planar shapes. Pattern Recognition, 20(3):262-272, 1987.

2. Robert M. Haralick, K. Shanmugam, and Itshak Dinstein. Textural features for image classification. IEEE Transactions on Systems, Man, and Cybernetics, SMC6(6):610-621, November 1973 .

3. Chih-Cheng Hsu, Wesley W. Chu, and Ricky K. Taira. A knowledge-based approarh for retrieving images by content. IEEE Tronsactions on Knowledge and. Dato Endancernng, 8(1):522-532, Angust 1996 .

4. Ming-Kei Hu. Visual pattern recognition by moment invariants. IRE Transactions on Information Theory, 8(2):179-187, 1962.

5. Markus Peura and Jukka Iivarinen. Efficiency of simple shape descriptors. In IWVF3, 3th International Workshop on Visual Forms, Capri, Italy, May 1997.

6. Milan Sonka, Vaclav Hlavac, and Roger Boyle Image Processing, Anulysis and Machne. Vqson. Chapman-Hall Computing, London, 1995. 